\title{
Association Between Resource Utilization and Patient Satisfaction at a Tertiary Care Medical Center
}

\author{
Eric A. Biondi, MD, MSBA ${ }^{1 \star}$, Matthew Hall, $\mathrm{PhD}^{2}$, Michael S. Leonard, MD, MS ${ }^{3}$, Paul A. Pirraglia, MD, MPH${ }^{4}$, \\ Brian K. Alverson, MD ${ }^{5}$
}

\begin{abstract}
${ }^{1}$ Department of Pediatrics, University of Rochester Medical Center, Rochester, New York; ${ }^{2}$ Children's Hospital Association, Overland Park, Kansas; ${ }^{3}$ Department of Pediatrics and Department of Public Health Sciences, University of Rochester Medical Center, Rochester, New York; ${ }^{4}$ Providence Veteran's Affairs Medical Center and Alpert Medical School of Brown University, Providence, Rhode Island; ${ }^{5}$ Department of Pediatrics, Rhode Island Hospital, Providence, Rhode Island.
\end{abstract}

BACKGROUND: The Centers for Medicare and Medicaid Services has emphasized patient satisfaction as a means by which hospitals should be compared and as a component of financial reimbursement. We sought to identify whether resource utilization is associated with patient satisfaction ratings.

DESIGN: This was a retrospective, cohort study over a 27month period from January 2012 to April 2014 of adult respondents ( $n=10,007$ ) to the Hospital Consumer Assessment of Healthcare Providers and Systems survey at a tertiary care medical center. For each returned survey, we developed a resource intensity score related to the corresponding hospitalization. We calculated a raw satisfaction rating (RSR) for each returned survey. Multivariable logistic regression was used to determine the association between resource intensity and top decile RSRs, using those with the lowest resource intensity as the reference group.
RESULTS: Adjusting for age, gender, insurance payer, severity of illness, and clinical service, patients in higher resource intensity groups were more likely to assign top decile RSRs than the lowest resource intensity group ("moderate" [adjusted odds ratio $\{$ aOR $\}$ : 1.42, 95\% confidence interval $\{\mathrm{Cl}\}$ : 1.11-1.83], "major" [aOR: 1.56, 95\% Cl: 1.22-2.01], and "extreme" [aOR: 2.29, 95\% Cl: 1.8-2.92]).

CONCLUSIONS: Resource utilization may be positively associated with patient satisfaction. These data suggest that hospitals with higher per-patient expenditures may receive higher ratings, which could result in hospitals with higher per-patient resource utilization appearing more attractive to healthcare consumers. Journal of Hospital Medicine 2016;11:785-791. (c) 2016 Society of Hospital Medicine
The patient experience has become increasingly important to healthcare in the United States. It is now a metric used commonly to determine physician compensation and accounts for nearly $30 \%$ of the Centers for Medicare and Medicaid Services' (CMS) Value-Based Purchasing (VBP) reimbursement for fiscal years 2015 and 2016. ${ }^{1,2}$

In April 2015, CMS added a 5-star patient experience score to its Hospital Compare website in an attempt to address the Affordable Care Act's call for transparent and easily understandable public reporting. ${ }^{3}$ A hospital's principal score is the Summary Star Rating, which is based on responses to the Hospital Consumer Assessment of Healthcare Providers and Systems (HCAHPS) survey. The formulas used to calculate Summary Star Ratings have been reported by CMS. ${ }^{4}$

Studies published over the past decade suggest that gender, age, education level, length of hospital stay,

*Address for correspondence and reprint requests: Eric Biondi, MD, 601 Elmwood Avenue, Box 667, Rochester NY, 14626; Telephone: 585-276-4113; Fax: 585-276-1128; E-mail: eric_biondi@urmc.rochester. edu

Additional Supporting Information may be found in the online version of this article.

Received: October 29, 2015; Revised: April 26, 2016; Accepted: May 8 , 2016

2016 Society of Hospital Medicine DOI 10.1002/jhm.2621

Published online in Wiley Online Library (Wileyonlinelibrary.com). travel distance, and other factors may influence patient satisfaction. ${ }^{5-8}$ One study utilizing a national dataset suggested that higher patient satisfaction was associated with greater inpatient healthcare utilization and higher healthcare expenditures. ${ }^{9}$ It is therefore possible that emphasizing patient experience scores could adversely impact healthcare resource utilization. However, positive patient experience may also be an important independent dimension of quality for patients and correlate with improved clinical outcomes. ${ }^{10}$

We know of no literature describing patient factors associated with the Summary Star Rating. Given that this rating is now used as a standard metric by which patient experience can be compared across more than 3,500 hospitals, ${ }^{11}$ data describing the association between patientlevel factors and the Summary Star Rating may provide hospitals with an opportunity to target improvement efforts. We aimed to determine the degree to which resource utilization is associated with a satisfaction score based on the Summary Star Rating methodology.

\section{METHODS}

The study was conducted at the University of Rochester Medical Center (URMC), an 830-bed tertiary care center in upstate New York. This was a retrospective review of all HCAHPS surveys returned to URMC over a 27-month period from January 1, 2012 to April 1, 2014. URMC follows the standard CMS 
process for determining which patients receive surveys as follows. During the study timeframe, HCAHPS surveys were mailed to patients 18 years of age and older who had an inpatient stay spanning at least 1 midnight. Surveys were mailed within 5 days of discharge, and were generally returned within 6 weeks. URMC did not utilize telephone or email surveys during the study period. Surveys were not sent to patients who (1) were transferred to another facility, (2) were discharged to hospice, (3) died during the hospitalization, (4) received psychiatric or rehabilitative services during the hospitalization, (5) had an international address, and/or (6) were prisoners.

The survey vendor (Press Ganey, South Bend, IN) for URMC provided raw data for returned surveys with patient answers to questions. Administrative and billing databases were used to add demographic and clinical data for the corresponding hospitalization to the dataset. These data included age, gender, payer status (public, private, self, charity), length of stay, number of attendings who saw the patient (based on encounters documented in the electronic medical record (EMR)), all discharge International Classification of Diseases, 9th Revision (ICD-9) diagnoses for the hospitalization, total charges for the hospitalization, and intensive care unit (ICU) utilization as evidenced by a documented encounter with a member of the Division of Critical Care/Pulmonary Medicine.

CMS analyzes surveys within 1 of 3 clinical service categories (medical, surgical, or obstetrics/gynecology) based on the discharging service. To parallel this approach, each returned survey was placed into 1 of these categories based on the clinical service of the discharging physician. Patients placed in the obstetrics/ gynecology category $(\mathrm{n}=1317,13 \%)$ will be analyzed in a future analysis given inherent differences in patient characteristics that require evaluation of other variables.

\section{Approximations of CMS Summary Star Rating}

The HCAHPS survey is a multiple-choice questionnaire that includes several domains of patient satisfaction. Respondents are asked to rate areas of satisfaction with their hospital experience on a Likert scale. CMS uses a weighted average of Likert responses to a subset of HCAHPS questions to calculate a hospital's raw score in 11 domains, as well as an overall raw summary score. CMS then adjusts each raw score for differences between hospitals (eg, clustering, improvement over time, method of survey) to determine a hospital's star rating in each domain and an overall Summary Star Rating (the Summary Star Rating is the primary factor by which consumers can compare hospitals). ${ }^{4}$ Because our data were from a single hospital system, the between-hospital scoring adjustments utilized by CMS were not applicable. Instead, we calculated the raw scores exactly as CMS does prior to the adjustments. Thus, our scores reflect the scores that CMS would have given URMC during the study period prior to standardized adjustments; we refer to this as the raw satisfaction rating (RSR). We calculated an RSR for every eligible survey. The RSR was calculated as a continuous variable from 0 (lowest) to 1 (highest). Detailed explanation of our RSR calculation is available in the Supporting Information in the online version of this article.

\section{Statistical Analysis}

All analyses were performed in aggregate and by service (medical vs surgical). Categorical variables were summarized using frequencies with percentages. Comparisons across levels of categorical variables were performed with the $\chi^{2}$ test. We report bivariate associations between the independent variables and RSRs in the top decile using unadjusted odds ratios (ORs) with $95 \%$ confidence intervals (CIs). Similarly, multivariable logistic regression was used for adjusted analyses. For the variables of severity of illness and resource intensity, the group with the lowest illness severity and lowest resource use served as the reference groups. We modeled patients without an ICU encounter and with an ICU encounter separately.

Charges, number of unique attendings encountered, and lengths of stay were highly correlated, and likely various measures of the same underlying construct of resource intensity, and therefore could not be entered into our models simultaneously. We combined these into a resource intensity score using factor analysis with a varimax rotation, and extracted factor scores for a single factor (supported by a scree plot). We then placed patients into 4 groups based on the distribution of the factor scores: low ( $<25$ th percentile), moderate (25th-50th percentile), major (50th-75th percentile), and extreme ( $>75$ th percentile).

We used the Charlson-Deyo comorbidity score as our disease severity index. ${ }^{12}$ The index uses ICD-9 diagnoses with points assigned for the impact of each diagnosis on morbidity and the points summed to an overall score. This provides a measure of disease severity for a patient based on the number of diagnoses and relative mortality of the individual diagnoses. Scores were categorized as 0 (representing no major illness burden), 1 to 3,4 to 6 , and $>6$.

All statistical analyses were performed using SAS version 9.4 (SAS Institute, Cary, NC), and P values $<0.05$ were considered statistically significant. This study was approved by the institutional review board at the University of Rochester Medical Center.

\section{RESULTS}

Our initial search identified 10,007 returned surveys (29\% of eligible patients returned surveys during the study period). Of these, $5059(51 \%)$ were categorized as medical, $3630(36 \%)$ as surgical, and $1317(13 \%)$ as obstetrics/gynecology. One survey did not have the service of the discharging physician recorded and was excluded. Cohort demographics and relationship to 
TABLE 1. Cohort Demographics and Raw Satisfaction Ratings in the Top Decile

\begin{tabular}{|c|c|c|c|c|c|c|c|c|c|c|c|c|}
\hline & \multicolumn{4}{|c|}{ Overall } & \multicolumn{4}{|c|}{ Medical } & \multicolumn{4}{|c|}{ Surgical } \\
\hline & Total & $<90$ th & Top Decile & $P$ & Total & $<90$ th & Top Decile & $P$ & Total & $<90$ th & Top Decile & $P$ \\
\hline Overall & 8,689 & $7,789(90)$ & $900(10)$ & & 5,059 & $4,646(92)$ & $413(8)$ & & 3,630 & $3,143(87)$ & $487(13)$ & \\
\hline \multicolumn{13}{|l|}{ Age, y } \\
\hline$<30$ & $419(5)$ & $371(89)$ & 48 (12) & $<0.001$ & $218(4)$ & $208(95)$ & $10(5)$ & $<0.001$ & $201(6)$ & $163(81)$ & $38(19)$ & $<0.001$ \\
\hline $30-49$ & $1,029(12)$ & $902(88)$ & $127(12)$ & & $533(11)$ & $482(90)$ & $51(10)$ & & 496 (14) & $420(85)$ & $76(15)$ & \\
\hline $50-69$ & $3,911(45)$ & $3,450(88)$ & 461 (12) & & $2,136(42)$ & $1,930(90)$ & $206(10)$ & & $1,775(49)$ & $1,520(86)$ & $255(14)$ & \\
\hline$>69$ & $3,330(38)$ & 3,066 (92) & $264(8)$ & & $2,172(43)$ & $2,026(93)$ & $146(7)$ & & $1,158(32)$ & $1,040(90)$ & $118(10)$ & \\
\hline \multicolumn{13}{|l|}{ Gender } \\
\hline Male & $4,640(53)$ & $4,142(89)$ & $498(11)$ & 0.220 & $2,596(51)$ & $2,379(92)$ & $217(8)$ & 0.602 & $2,044(56)$ & $1,763(86)$ & $281(14)$ & 0.506 \\
\hline Female & $4,049(47)$ & $3,647(90)$ & $402(10)$ & & $2,463(49)$ & $2,267(92)$ & $196(8)$ & & $1,586(44)$ & $1,380(87)$ & $206(13)$ & \\
\hline \multicolumn{13}{|l|}{ ICU encounter } \\
\hline No & $7,122(82)$ & $6,441(90)$ & $681(10)$ & $<0.001$ & $4,547(90)$ & $4,193(92)$ & $354(8)$ & $<0.001$ & $2,575(71)$ & $2,248(87)$ & $327(13)$ & 0.048 \\
\hline Yes & $1,567(18)$ & $1,348(86)$ & $219(14)$ & & $512(10)$ & $453(89)$ & $59(12)$ & & $1,055(29)$ & $895(85)$ & $160(15)$ & \\
\hline \multicolumn{13}{|l|}{ Payer } \\
\hline Public & $5,564(64)$ & $5,036(91)$ & $528(10)$ & $<0.001$ & $3,424(68)$ & $3,161(92)$ & $263(8)$ & 0.163 & $2,140(59)$ & $1,875(88)$ & 265 (12) & 0.148 \\
\hline Private & $3,064(35)$ & $2,702(88)$ & $362(12)$ & & $1,603(32)$ & $1,458(91)$ & $145(9)$ & & $1,461(40)$ & $1,244(85)$ & $217(15)$ & \\
\hline Charity & 45 (1) & 37 (82) & $8(18)$ & & $25(1)$ & $21(84)$ & $4(16)$ & & 20 (1) & $16(80)$ & $4(20)$ & \\
\hline Self & $16(0)$ & $14(88)$ & $2(13)$ & & $7(0)$ & $6(86)$ & $1(14)$ & & $9(0)$ & $8(89)$ & $1(11)$ & \\
\hline \multicolumn{13}{|l|}{ Length of stay, d } \\
\hline$<3$ & $3,156(36)$ & $2,930(93)$ & $226(7)$ & $<0.001$ & $1,961(39)$ & $1,865(95)$ & $96(5)$ & $<0.001$ & $1,195(33)$ & $1,065(89)$ & $130(11)$ & $<0.001$ \\
\hline $3-6$ & $3,330(38)$ & $2,959(89)$ & $371(11)$ & & $1,867(37)$ & $1,702(91)$ & $165(9)$ & & $1,463(40)$ & $1,257(86)$ & $206(14)$ & \\
\hline$>6$ & $2,203(25)$ & $1,900(86)$ & $303(14)$ & & $1,231(24)$ & $1,079(88)$ & $152(12)$ & & $972(27)$ & $821(85)$ & $151(16)$ & \\
\hline \multicolumn{13}{|l|}{ No. of attendings } \\
\hline$<4$ & $3,959(46)$ & $3,615(91)$ & $344(9)$ & $<0.001$ & $2,307(46)$ & $2,160(94)$ & $147(6)$ & $<0.001$ & $1,652(46)$ & $1,455(88)$ & 197 (12) & 0.052 \\
\hline $4-6$ & $3,067(35)$ & $2,711(88)$ & 356 (12) & & $1,836(36)$ & $1,663(91)$ & $173(9)$ & & $1,231(34)$ & $1,048(85)$ & $183(15)$ & \\
\hline$>6$ & $1,663(19)$ & $1,463(88)$ & 200 (12) & & $916(18)$ & $823(90)$ & $93(10)$ & & 747 (21) & $640(86)$ & 107 (14) & \\
\hline \multicolumn{13}{|l|}{ Severity index* } \\
\hline 0 (lowest) & $2,812(32)$ & $2,505(89)$ & 307 (11) & 0.272 & $1,273(25)$ & $1,185(93)$ & $88(7)$ & 0.045 & $1,539(42)$ & $1,320(86)$ & $219(14)$ & 0.261 \\
\hline $1-3$ & $4,253(49)$ & $3,827(90)$ & $426(10)$ & & $2,604(52)$ & $2,395(92)$ & $209(8)$ & & $1,649(45)$ & $1,432(87)$ & $217(13)$ & \\
\hline $4-6$ & $1163(13)$ & $1,052(91)$ & $111(10)$ & & 849 (17) & $770(91)$ & $79(9)$ & & $314(9)$ & $282(90)$ & $32(10)$ & \\
\hline$>6$ (highest) & $461(5)$ & $405(88)$ & $56(12)$ & & $333(7)$ & $296(89)$ & 37 (11) & & $128(4)$ & $109(85)$ & $19(15)$ & \\
\hline \multicolumn{13}{|l|}{ Charges, ${ }^{\dagger}$} \\
\hline Low & $1,820(21)$ & $1,707(94)$ & $113(6)$ & $<0.001$ & $1,426(28)$ & $1,357(95)$ & $69(5)$ & $<0.001$ & 394 (11) & $350(89)$ & $44(11)$ & 0.007 \\
\hline Medium & $5,094(59)$ & 4,581 (90) & $513(10)$ & & $2,807(56)$ & $2,582(92)$ & $225(8)$ & & $2,287(63)$ & $1,999(87)$ & $288(13)$ & \\
\hline High & $1,775(20)$ & $1,501(85)$ & $274(15)$ & & $826(16)$ & $707(86)$ & $119(14)$ & & $949(26)$ & 794 (84) & $155(16)$ & \\
\hline
\end{tabular}

NOTE: Data are presented as no. (\%). Percentages may not add up to 100 due to rounding. Abbreviations: ICU, intensive care unit. ${ }^{*}$ Calculated using the Charlson-Deyo index; smaller values indicate less severity. ${ }^{\dagger}$ Low $=\langle \$ 10,000$; medium $=\$ 10,000-\$ 40,000$; high $=>\$ 40,000$.

RSRs in the top decile for the 8689 medical and surgical patients can be found in Table 1 . The most common discharge diagnosis-related groups (DRGs) for medical patients were 247, percutaneous cardiovascular procedure with drug-eluding stent without major complications or comorbidities (MCC) $(3.8 \%)$; 871, septicemia or severe sepsis without mechanical ventilation >96 hours with MCC $(2.7 \%)$; and 392, esophagitis, gastroenteritis, and miscellaneous digestive disorders with MCC $(2.3 \%)$. The most common DRGs for surgical patients were 460, spinal fusion except cervical without MCC $(3.5 \%)$; 328, stomach, esophageal and duodenal procedure without complication or comorbidities or MCC (3.3\%); and 491, back and neck procedure excluding spinal fusion without complication or comorbidities or MCC $(3.1 \%)$.

Unadjusted analysis of medical and surgical patients identified significant associations of several variables with a top decile RSR (Table 2). Patients with longer lengths of stay (OR: 2.07, 95\% CI: 1.72-2.48), more attendings (OR: 1.44, 95\% CI: 1.19-1.73), and higher hospital charges (OR: 2.76, 95\% CI: 2.19-3.47) were more likely to report an RSR in the top decile. Patients without an ICU encounter (OR: 0.65, 95\% CI: $0.55-0.77$ ) and on a medical service (OR: $0.57,95 \%$ CI: 0.5- 0.66) were less likely to report an RSR in the top decile. Several associations were identified in only the medical or surgical cohorts. In the medical cohort, patients with the highest illness severity index (OR: 1.68, 95\% CI: 1.12- 2.52) and with $\geq 7$ different attending physicians (OR: 1.66, 95\% CI: $1.27-2.18$ ) were more likely to report RSRs in the top decile. In the surgical cohort, patients $<30$ years of age (OR: $2.05,95 \%$ CI 1.38-3.07) were more likely to report an RSR in the top decile than patients $>69$ years of age. Insurance payer category and gender were not significantly associated with top decile RSRs.

Multivariable modeling (Table 3) for all patients without an ICU encounter suggested that (1) patients aged $<30$ years, 30 to 49 years, and 50 to 69 years were more likely to report top decile RSRs when compared to patients 70 years and older (OR: 1.61, 95\% 
TABLE 2. Bivariate Comparisons of Associations Between Top Decile Satisfaction Ratings and Reference Levels

\begin{tabular}{|c|c|c|c|c|c|c|}
\hline & \multicolumn{2}{|c|}{ Overall } & \multicolumn{2}{|c|}{ Medical } & \multicolumn{2}{|c|}{ Surgical } \\
\hline & Odds Ratio (95\% Cl) & $P$ & Odds Ratio $(95 \% \mathrm{Cl})$ & $P$ & Odds Ratio $(95 \% \mathrm{Cl})$ & $P$ \\
\hline \multicolumn{7}{|l|}{ Age, y } \\
\hline$<30$ & $1.5(1.08-2.08)$ & 0.014 & $0.67(0.35-1.29)$ & 0.227 & $2.05(1.38-3.07)$ & $<0.001$ \\
\hline $30-49$ & $1.64(1.31-2.05)$ & $<.001$ & $1.47(1.05-2.05)$ & 0.024 & $1.59(1.17-2.17)$ & 0.003 \\
\hline $50-69$ & $1.55(1.32-1.82)$ & $<.001$ & $1.48(1.19-1.85)$ & 0.001 & $1.48(1.17-1.86)$ & 0.001 \\
\hline$>69$ & \multicolumn{2}{|l|}{ Ref } & \multicolumn{2}{|l|}{ Ref } & \multicolumn{2}{|l|}{ Ref } \\
\hline \multicolumn{7}{|l|}{ Gender } \\
\hline Male & $1.09(0.95-1.25)$ & 0.220 & $1.06(0.86-1.29)$ & 0.602 & $1.07(0.88-1.3)$ & 0.506 \\
\hline Female & \multicolumn{2}{|l|}{ Ref } & \multicolumn{2}{|l|}{ Ref } & \multicolumn{2}{|l|}{ Ref } \\
\hline \multicolumn{7}{|l|}{ ICU encounter } \\
\hline № & $0.65(0.55-0.77)$ & $<0.001$ & $0.65(0.48-0.87)$ & 0.004 & $0.81(0.66-1)$ & 0.048 \\
\hline Yes & \multicolumn{2}{|l|}{ Ref } & \multicolumn{2}{|l|}{ Ref } & \multicolumn{2}{|c|}{ Ref } \\
\hline \multicolumn{7}{|l|}{ Payer } \\
\hline Public & $0.73(0.17-3.24)$ & 0.683 & $0.5(0.06-4.16)$ & 0.521 & $1.13(0.14-9.08)$ & 0.908 \\
\hline Private & $0.94(0.21-4.14)$ & 0.933 & $0.6(0.07-4.99)$ & 0.634 & $1.4(0.17-11.21)$ & 0.754 \\
\hline Charity & $1.51(0.29-8.02)$ & 0.626 & $1.14(0.11-12.25)$ & 0.912 & $2(0.19-20.97)$ & 0.563 \\
\hline Self & \multicolumn{2}{|l|}{ Ref } & \multicolumn{2}{|l|}{ Ref } & \multicolumn{2}{|l|}{ Ref } \\
\hline \multicolumn{7}{|l|}{ Length of stay, d } \\
\hline$<3$ & \multicolumn{2}{|c|}{ Ref } & \multicolumn{2}{|c|}{ Ref } & \multicolumn{2}{|c|}{ Ref } \\
\hline $3-6$ & $1.63(1.37-1.93)$ & $<0.001$ & $1.88(1.45-2.44)$ & $<0.001$ & $1.34(1.06-1.7)$ & 0.014 \\
\hline$>6$ & $2.07(1.72-2.48)$ & $<0.001$ & $2.74(2.1-3.57)$ & $<0.001$ & $1.51(1.17-1.94)$ & 0.001 \\
\hline \multicolumn{7}{|l|}{ No. of attendings } \\
\hline$<4$ & \multicolumn{2}{|c|}{ Ref } & \multicolumn{2}{|c|}{ Ref } & \multicolumn{2}{|c|}{ Ref } \\
\hline $4-6$ & $1.38(1.18-1.61)$ & $<0.001$ & $1.53(1.22-1.92)$ & $<0.001$ & $1.29(1.04-1.6)$ & 0.021 \\
\hline$>6$ & $1.44(1.19-1.73)$ & $<0.001$ & $1.66(1.27-2.18)$ & $<0.001$ & $1.23(0.96-1.59)$ & 0.102 \\
\hline Severity index* & & & & & & \\
\hline 0 (lowest) & Ref & & $\operatorname{Re}$ & & Ref & \\
\hline $1-3$ & $0.91(0.78-1.06)$ & 0.224 & $1.18(0.91-1.52)$ & 0.221 & $0.91(0.75-1.12)$ & 0.380 \\
\hline $4-6$ & $0.86(0.68-1.08)$ & 0.200 & $1.38(1.01-1.9)$ & 0.046 & $0.68(0.46-1.01)$ & 0.058 \\
\hline$>6$ (highest) & $1.13(0.83-1.53)$ & 0.436 & $1.68(1.12-2.52)$ & 0.012 & $1.05(0.63-1.75)$ & 0.849 \\
\hline Charges $\dagger$ & & & & & & \\
\hline Low & Ref & & $\mathrm{Re}$ & & Ref & \\
\hline Medium & $1.69(1.37-2.09)$ & $<0.001$ & $1.71(1.3-2.26)$ & $<0.001$ & $1.15(0.82-1.61)$ & 0.428 \\
\hline High & $2.76(2.19-3.47)$ & $<0.001$ & $3.31(2.43-4.51)$ & $<0.001$ & 1.55 (1.09-2.22) & 0.016 \\
\hline Service & & & & & & \\
\hline Medical & $0.57(0.5-0.66)$ & $<0.001$ & & & & \\
\hline Surgical & Ref & & & & & \\
\hline
\end{tabular}

NOTE: Abbreviations: $\mathrm{Cl}$, confidence interval; ICU, intensive care unit; Ref, reference. ${ }^{*}$ Calculated using the Charlson-Deyo index; smaller values indicate less severity. $\dagger$ Low $=\langle \$ 10,000 ;$ medium $=\$ 10,000-\$ 40,000 ;$ high $=$ $>\$ 40,000$.

CI: 1.09-2.36; OR: 1.44, 95\% CI: 1.08-1.93; and OR: $1.39,95 \%$ CI: $1.13-1.71$, respectively) and (2), when compared to patients with extreme resource intensity scores, patients with higher resource intensity scores were more likely to report top decile RSRs (moderate [OR: 1.42, 95\% CI: 1.11-1.83], major [OR: 1.56, 95\% CI: 1.22-2.01], and extreme [OR: $2.29,95 \%$ CI: 1.8-2.92]. These results were relatively consistent within medical and surgical subgroups (Table 3).

In those with at least $1 \mathrm{ICU}$ attending encounter (see Supporting Table 1 in the online version of this article), no variables demonstrated significant association with top decile RSRs in the overall group or in the medical subgroup. For surgical patients with at least 1 ICU attending encounter (see Supporting Table 1 in the online version of this article), patients aged 30 to 49 and 50 to 69 years were more likely to provide top decile RSRs (OR: 1.93, 95\% CI: 1.08-3.46 and OR: 1.65, $95 \%$ CI 1.07-2.53, respectively). Resource intensity was not significantly associated with top decile RSRs.

\section{DISCUSSION}

Our analysis suggests that, for patients on the general care floors, resource utilization is associated with the RSR and, therefore, potentially the CMS Summary Star Rating. Adjusting for severity of illness, patients with higher resource utilization were more likely to report top decile RSRs.

Prior data regarding utilization and satisfaction are mixed. In a 2-year, prospective, national examination, patients in the highest quartile of patient satisfaction had increased healthcare and prescription drug expenditures as well as increased rates of hospitalization when compared with patients in the lowest quartile of patient satisfaction. ${ }^{9}$ However, a recent national study of surgical administrative databases suggested hospitals with high patient satisfaction provided more efficient care. ${ }^{13}$

One reason for the conflicting data may be that large, national evaluations are unable to control for between-hospital confounders (ie, hospital quality of 
TABLE 3. Multivariable Logistic Regression Model for Top Decile Raw Satisfaction Ratings for Patients on the General Wards*

\begin{tabular}{|c|c|c|c|c|c|c|}
\hline & \multicolumn{2}{|c|}{ Overall } & \multicolumn{2}{|c|}{ Medical } & \multicolumn{2}{|c|}{ Surgical } \\
\hline & Odds Ratio (95\% Cl) & $P$ & Odds Ratio (95\% Cl) & $P$ & Odds Ratio (95\% Cl) & $P$ \\
\hline \multicolumn{7}{|l|}{ Age,y } \\
\hline$<30$ & $1.61(1.09-2.36)$ & 0.016 & $0.82(0.4-1.7)$ & 0.596 & $2.31(1.39-3.82)$ & 0.001 \\
\hline $30-49$ & $1.44(1.08-1.93)$ & 0.014 & $1.55(1.03-2.32)$ & 0.034 & $1.41(0.91-2.17)$ & 0.120 \\
\hline $50-69$ & $1.39(1.13-1.71)$ & 0.002 & $1.44(1.1-1.88)$ & 0.008 & $1.39(1-1.93)$ & 0.049 \\
\hline$>69$ & Ref & & Ref & & Ref & \\
\hline \multicolumn{7}{|l|}{ Sex } \\
\hline Male & $1(0.85-1.17)$ & 0.964 & $1(0.8-1.25)$ & 0.975 & $0.99(0.79-1.26)$ & 0.965 \\
\hline Female & Ref & & Ref & & Ref & \\
\hline \multicolumn{7}{|l|}{ Payer } \\
\hline Public & $0.62(0.14-2.8)$ & 0.531 & $0.42(0.05-3.67)$ & 0.432 & $1.03(0.12-8.59)$ & 0.978 \\
\hline Private & $0.67(0.15-3.02)$ & 0.599 & $0.42(0.05-3.67)$ & 0.434 & $1.17(0.14-9.69)$ & 0.884 \\
\hline Charity & $1.54(0.28-8.41)$ & 0.620 & $1(0.09-11.13)$ & 0.999 & $2.56(0.23-28.25)$ & 0.444 \\
\hline Self & Ref & & Ref & & Ref & \\
\hline \multicolumn{7}{|l|}{ Severity index $x_{\dagger}$} \\
\hline 0 (lowest) & Ref & & Ref & & Ref & \\
\hline $1-3$ & $1.07(0.89-1.29)$ & 0.485 & $1.18(0.88-1.58)$ & 0.267 & $1(0.78-1.29)$ & 0.986 \\
\hline $4-6$ & $1.14(0.86-1.51)$ & 0.377 & $1.42(0.99-2.04)$ & 0.056 & $0.6(0.33-1.1)$ & 0.100 \\
\hline$>6$ (highest) & $1.31(0.91-1.9)$ & 0.150 & $1.47(0.93-2.33)$ & 0.097 & $1.1(0.54-2.21)$ & 0.795 \\
\hline \multicolumn{7}{|l|}{ Resource intensity scorę } \\
\hline Low & Ref & & Ref & & Ref & \\
\hline Moderate & $1.42(1.11-1.83)$ & 0.006 & $1.6(1.11-2.3)$ & 0.011 & $0.94(0.66-1.34)$ & 0.722 \\
\hline Major & $1.56(1.22-2.01)$ & 0.001 & $1.69(1.18-2.43)$ & 0.004 & $1.28(0.91-1.8)$ & 0.151 \\
\hline Extreme & $2.29(1.8-2.92)$ & $<0.001$ & $2.72(1.94-3.82)$ & $<0.001$ & $1.63(1.17-2.26)$ & 0.004 \\
\hline \multicolumn{7}{|l|}{ Service } \\
\hline Medical & $0.59(0.5-0.69)$ & $<0.001$ & & & & \\
\hline Surgical & Ref & & & & & \\
\hline
\end{tabular}

NOTE: Abbreviations: $\mathrm{Cl}$, confidence interval; Ref, reference. ${ }^{\star}$ Excludes the 1,567 patients who had an intensive care unit encounter. †Calculated using the Charlson-Deyo index. $\ddagger$ Component variables include length of stay, number of attendings, and charges

care). By capturing all eligible returned surveys at 1 institution, our design allowed us to collect granular data. We found that in 1 hospital setting, patient population, facilities, and food services, patients receiving more clinical resources generally assigned higher ratings than patients receiving less.

It is possible that utilization is a proxy for serious illness, and that patients with serious illness receive more attention during hospitalization and are more satisfied when discharged in a good state of health. However, we did adjust for severity of illness in our model using the Charlson-Deyo index and we suggest that, other factors being equal, hospitals with higher per-patient expenditures may be assigned higher Summary Star Ratings.

CMS has recently implemented a number of metrics designed to decrease healthcare costs by improving quality, safety, and efficiency. Concurrently, CMS has also prioritized patient experience. The Summary Star Rating was created to provide healthcare consumers with an easy way to compare the patient experience between hospitals ${ }^{4}$; however, our data suggest that this metric may be at odds with inpatient cost savings and efficiency metrics.

Per-patient spending becomes particularly salient when considering that in fiscal year 2016, CMS' hospital VBP reimbursement will include 2 metrics: an efficiency outcome measure labeled "Medicare spending per beneficiary," and a patient experience outcome measure based on HCAHPS survey dimensions. ${ }^{2}$ Together, these 2 metrics will comprise nearly half of the total VBP performance score used to determine reimbursement. Although our data suggest that these 2 VBP metrics may be correlated, it should be noted that we measured inpatient hospital charges, whereas the CMS efficiency outcome measure includes costs for episode of care spanning 3 days prior to hospitalization to 30 days after hospitalization.

Patient expectations likely play a role in satisfaction. ${ }^{14-16}$ In an outpatient setting, physician fulfillment of patient requests has been associated with positive patient evaluations of care ${ }^{17}$ However, patients appear to value education, shared decision making, and provider empathy more than testing and intervention. ${ }^{14,18-23}$ Perhaps, in the absence of the former attributes, patients use additional resource expenditure as a proxy.

It is not clear that higher resource expenditure improves outcomes. A landmark study of nearly 1 million Medicare enrollees by Fisher et al. suggests that, although Medicare patients in higher-spending regions receive more care than those in lower-spending regions, this does not result in better health outcomes, specifically with regard to mortality. ${ }^{24,25}$ Patients who live in areas of high hospital capacity use the hospital more 
frequently than do patients in areas of low hospital capacity, but this does not appear to result in improved mortality rates. ${ }^{26}$ In fact, physicians in areas of high healthcare capacity report more difficulty maintaining high-quality patient relationships and feel less able to provide high-quality care than physicians in lowercapacity areas. $^{27}$

We hypothesize the cause of the association between resource utilization and patient satisfaction could be that patients (1) perceive that a doctor who allows them to stay longer in the hospital or who performs additional testing cares more about their wellbeing and (2) that these patients feel more strongly that their concerns are being heard and addressed by their physicians. A systematic review of primary care patients identified many studies that found a positive association between meeting patient expectations and satisfaction with care, but also suggested that although patients frequently expect information, physicians misperceive this as an expectation of specific action. ${ }^{28}$ A separate systematic review found that patient education in the form of decision aides can help patients develop more reasonable expectations and reduce utilization of certain discretionary procedures such as elective surgeries and prostate-specific antigen testing. ${ }^{29}$

We did not specifically address clinical outcomes in our analysis because the clinical outcomes on which CMS currently adjusts VBP reimbursement focus on 30-day mortality for specific diagnoses, nosocomial infections, and iatrogenic events. ${ }^{30}$ Our data include only returned surveys from living patients, and it is likely that 30-day mortality was similar throughout all subsets of patients. Additionally, the nosocomial and iatrogenic outcome measures used by CMS are sufficiently rare on the general floors and are unlikely to have significantly influenced our results. ${ }^{31}$

Our study has several strengths. Nearly all medical and surgical patient surveys returned during the study period were included, and therefore our calculations are likely to accurately reflect the Summary Star Rating that would have been assigned for the period. Second, the large sample size helps attenuate potential differences in commonly used outcome metrics. Third, by adjusting for a variety of demographic and clinical variables, we were able to decrease the likelihood of unidentified confounders.

Notably, we identified $38(0.4 \%)$ surveys returned for patients under 18 years of age at admission. These surveys were included in our analysis because, to the best of our knowledge, they would have existed in the pool of surveys CMS could have used to assign a Summary Star Rating.

Our study also has limitations. First, geographically diverse data are needed to ensure generalizability. Second, we used the Charlson-Deyo Comorbidity Index to describe the degree of illness for each patient. This index represents a patient's total illness burden but may not describe the relative severity of the patient's current illness relative to another patient. Third, we selected variables we felt were most likely to be associated with patient experience, but unidentified confounding remains possible. Fourth, attendings caring for ICU patients fall within the Division of Critical Care/Pulmonary Medicine. Therefore, we may have inadvertently placed patients into the ICU cohort who received a pulmonary/critical care consult on the general floors. Fifth, our data describe associations only for patients who returned surveys. Although there may be inherent biases in patients who return surveys, HCAHPS survey responses are used by CMS to determine a hospital's overall satisfaction score.

\section{CONCLUSION}

For patients who return HCAHPS surveys, resource utilization may be positively associated with a hospital's Summary Star Rating. These data suggest that hospitals with higher per-patient expenditures may receive higher Summary Star Ratings, which could result in hospitals with higher per-patient resource utilization appearing more attractive to healthcare consumers. Future studies should attempt to confirm our findings at other institutions and to determine causative factors.

\section{Acknowledgements}

The authors thank Jason Machan, PhD (Department of Orthopedics and Surgery, Warren Alpert Medical School, Brown University, Providence, Rhode Island) for his help with study design, and Ms. Brenda Foster (data analyst, University of Rochester Medical Center, Rochester, NY) for her help with data collection.

Disclosures: Nothing to report.

\section{References}

1. Finkelstein J, Lifton J, Capone C. Redesigning physician compensation and improving ED performance. Healthc Financ Manage. 2011;65(6): 114-117.

2. QualityNet. Available at: https:/www.qualitynet.org/dcs/Content Server $? \mathrm{c}=$ Page $\&$ pagename $=$ QnetPublic $\% 2$ FPage $\% 2 F Q n e t T i e r 3 \&$ cid $=$ 1228772237147. Accessed October 5, 2015.

3. Centers for Medicare and Medicaid Services. Hospital Compare. Available at: https://www.medicare.gov/hospitalcompare/search.html. Accessed October 5, 2015.

4. Centers for Medicare and Medicaid Services. HCAHPS Star Ratings Technical Notes. Available at: http:/www.hcahpsonline.org/files/ hcahps_stars_tech_notes_october_2015.pdf. Accessed October 5, 2015.

5. Abtahi AM, Presson AP, Zhang C, Saltzman CL, Tyser AR. Association between orthopaedic outpatient satisfaction and non-modifiable patient factors. J Bone Joint Surg Am. 2015;97(13):1041-1048.

6. Nguyen Thi PL, Briancon S, Empereur F, Guillemin F. Factors determining inpatient satisfaction with care. Soc Sci Med. 2002;54(4):493-504.

7. Hekkert KD, Cihangir S, Kleefstra SM, van den Berg B, Kool RB. Patient satisfaction revisited: a multilevel approach. Soc Sci Med. 2009;69(1):68-75.

8. Quintana JM, Gonzalez N, Bilbao A, et al. Predictors of patient satisfaction with hospital health care. BMC Health Serv Res. 2006;6:102.

9. Fenton JJ, Jerant AF, Bertakis KD, Franks P. The cost of satisfaction: a national study of patient satisfaction, health care utilization, expenditures, and mortality. Arch Intern Med. 2012;172(5):405-411.

10. Boulding W, Glickman SW, Manary MP, Schulman KA, Staelin R. Relationship between patient satisfaction with inpatient care and hospital readmission within 30 days. Am J Manag Care. 2011;17(1): 41-48.

11. Becker's Infection Control and Clinical Quality. Star Ratings go live on Hospital Compare: how many hospitals got 5 stars? Available at: http://www.beckershospitalreview.com/quality/star-ratings-go-liveon-hospital-compare-how-many-hospitals-got-5-stars.html. Published April 16, 2015. Accessed October 5, 2015.

12. Deyo RA, Cherkin DC, Ciol MA. Adapting a clinical comorbidity index for use with ICD-9-CM administrative databases. J Clin Epidemiol. 1992;45(6):613-619. 
13. Tsai TC, Orav EJ, Jha AK. Patient satisfaction and quality of surgical care in US hospitals. Ann Surg. 2015;261(1):2-8.

14. Anhang Price R, Elliott MN, Cleary PD, Zaslavsky AM, Hays RD. Should health care providers be accountable for patients' care experiences? J Gen Intern Med. 2015;30(2):253-256.

15. Bell RA, Kravitz RL, Thom D, Krupat E, Azari R. Unmet expectations for care and the patient-physician relationship. I Gen Intern Med. 2002;17(11):817-824.

16. Peck BM, Ubel PA, Roter DL, et al. Do unmet expectations for specific tests, referrals, and new medications reduce patients' satisfaction? J Gen Intern Med. 2004;19(11):1080-1087.

17. Kravitz RL, Bell RA, Azari R, Krupat E, Kelly-Reif S, Thom D. Request fulfillment in office practice: antecedents and relationship to outcomes. Med Care. 2002;40(1):38-51.

18. Renzi C, Abeni D, Picardi A, et al. Factors associated with patient satisfaction with care among dermatological outpatients. Br J Dermatol. 2001;145(4):617-623.

19. Cooke T, Watt D, Wertzler W, Quan H. Patient expectations of emergency department care: phase II-a cross-sectional survey. CJEM. 2006;8(3):148-157.

20. Bendapudi NM, Berry LL, Frey KA, Parish JT, Rayburn WL. Patients' perspectives on ideal physician behaviors. Mayo Clin Proc. 2006; 81(3):338-344.

21. Wen LS, Tucker S. What do people want from their health care? A qualitative study. J Participat Med. 2015;18:e10.

22. Shah MB, Bentley JP, McCaffrey DJ III. Evaluations of care by adults following a denial of an advertisement-related prescription drug request: the role of expectations, symptom severity, and physician communication style. Soc Sci Med. 2006;62(4):888-899.
23. Paterniti DA, Fancher TL, Cipri CS, Timmermans S, Heritage J, Kravitz RL. Getting to "no": strategies primary care physicians use to deny patient requests. Arch Intern Med. 2010;170(4):381-388.

24. Fisher ES, Wennberg DE, Stukel TA, Gottlieb DJ, Lucas FL, Pinder EL. The implications of regional variations in Medicare spending. Part 1: the content, quality, and accessibility of care. Ann Intern Med. 2003;138(4):273-287.

25. Fisher ES, Wennberg DE, Stukel TA, Gottlieb DJ, Lucas FL, Pinder EL. The implications of regional variations in Medicare spending. Part 2: health outcomes and satisfaction with care. Ann Intern Med. $2003 ; 138(4): 288-298$.

26. Fisher ES, Wennberg JE, Stukel TA, et al. Associations among hospital capacity, utilization, and mortality of US Medicare beneficiaries, controlling for sociodemographic factors. Health Serv Res. 2000;34(6):1351-1362.

27. Sirovich BE, Gottlieb DJ, Welch HG, Fisher ES. Regional variations in health care intensity and physician perceptions of quality of care. Ann Intern Med. 2006;144(9):641-649.

28. Rao JK, Weinberger M, Kroenke K. Visit-specific expectations and patient-centered outcomes: a literature review. Arch Fam Med. 2000; 9(10):1148-1155.

29. Stacey D, Legare F, Col NF, et al. Decision aids for people facing health treatment or screening decisions. Cochrane Database Syst Rev. 2014;1:CD001431.

30. Centers for Medicare and Medicaid Services. Hospital Compare. Outcome domain. Available at: https://www.medicare.gov/hospitalcompare/data/outcome-domain.html. Accessed October 5, 2015.

31. Centers for Disease Control and Prevention. 2013 national and state healthcare-associated infections progress report. Available at: www. cdc.gov/hai/progress-report/index.html. Accessed October 5, 2015. 